\title{
Improved 3D Osteotomy Planning in Cranio-maxillofacial Surgery
}

\author{
Stefan Zachow ${ }^{1}$, Evgeny Gladilin ${ }^{1}$, Hans-Florian Zeilhofer ${ }^{2}$, and Robert Sader $^{2}$ \\ 1 Konrad-Zuse-Zentrum für Informationstechnik Berlin (ZIB) \\ http://www.zib.de/visual/projects/cas \\ ${ }^{2}$ Department of Oral \& Maxillofacial Surgery, University of Technology Munich
}

\begin{abstract}
In this paper we present two clinical cases in maxillofacial surgery, where complex surgical interventions have been pre-operatively planned on 3D models of the patients' heads. Our goal was to provide surgeons with an additional planning criterion, i.e. the prediction of the post-operative facial appearance. In our first study a two step mandibular distraction has been planned, and in the second one a bimaxillary operation with a high Le Fort I osteotomy of the maxilla according to Bell, as well as a sagittal split osteotomy on both sides of the mandible, according to Obwegeser-Dal Pont. Within our study we did focus on the three dimensional soft tissue simulation using finite element methods. For the provision of such a planning aid, concepts for an integrated 3D surgery planning system are proposed that are partially implemented and demonstrated.
\end{abstract}

Keywords: Computer-Assisted Cranio-Maxillofacial Surgery, Osteotomy, Osteodistraction, Soft Tissue Prediction, Finite-Element Methods

\section{Introduction}

The planning of complex osteotomies and multidirectional osteodistraction in craniomaxillofacial surgery requires a high degree of experience and expertise for retrieving an optimal rehabilitation. Live size skull facsimiles are very helpful, and currently an established and very valuable 3D planning aid. Soft tissue in its entirety, however, cannot be taken into account for pre-operative planning in clinical practice right now. Techniques for two dimensional profile analysis from lateral cephalograms including video overlays are giving a rough estimation on how the patient might look after an operation, but a full spatial prognosis of the patients' post-operative appearance is the most desired feature of an enhanced 3D planning system for computer assisted cranio-maxillofacial surgery.

First concepts of 3D planning in cranio-maxillofacial surgery including soft tissue prediction can be found in [1]. Active research started approximately 10 years ago [2, 3, 4]. Other research groups have continued working on the simulation of soft tissue deformation for maxillofacial surgery [5, 6]. To our knowledge, a surgical planning system, integrating all essential requirements for a sound clinical use does not yet exist. However, the combination of the latest results in this field of work is getting close to the envisioned goal [7, 8, 9, 10, 11]. 


\section{Material and Methods}

At our institute (ZIB) a prototype system has been developed that allows the planning of osteotomies including bone rearrangement on 3D patient models, derived from tomographic data. At the moment, our focus is on the prediction of the post-operative appearance by means of 3D soft tissue simulation [11]. The development platform is our 3D modeling and visualization system Amira [12]. A finite element approach on a tetrahedral grid of the entire facial soft tissue allows the prediction of the post-operative facial appearance, induced by the relocation of underlying bony structures [13, 14]. To improve our planning environment, a task level analysis lead to the following requirements for computer assisted 3D planning in maxillofacial surgery:

- New concepts for 3D cephalometry under provision of appropriate measuring tools and normative data are required [1, 10, 15.

- Tools for an intuitive definition of osteotomy lines on a 3D model of the skull are necessary (electronic pen, virtual saw).

- Drill, saw and plane operations must be incorporated into the planning. Material reduction has to be taken into account.

- Bony structures of the model have to be split or cut in consideration of vulnerable structures, like nerves and vessels. Individual parts have to be transformed quantifiably.

- 3D implant models (miniplates, screws and distraction devices) must be selectable from a toolbox. These items have to be positioned and fixed on the skull model in consideration of bone thickness and geometry. Planning aids for the assessment of optimal access paths or mount points are required [16].

- The prognosis of the patients' post-operative facial appearance induced by the planned rearrangement of bony structures has to become an additional aspect for pre-operative assessment of the surgical plan.

An improved 3D surgery planning system thus requires: a) techniques for the generation of adequate patient models, b) methods for intuitive and exact measurements and surgical manipulations on basis of such models, and c) the incorporation of fast and reliable soft tissue simulation into the planning.

\subsection{Modeling}

Prerequisite for the generation of anatomical correct volumetric patient models are tomographic data. An exact reconstruction of diverse tissue regions still requires semiautomatic techniques to avoid misclassification of fine structures or non-distinguishable tissue types. Appropriate tools for an efficient and accurate segmentation are provided with our planning environment as shown in figure 1

After classification of all relevant structures an automatic reconstruction of a topological correct, non-manifold surface model with subvoxel accuracy is generated [17, 18]. This model can be automatically simplified with arbitrary, adaptively chosen resolution to preserve relevant details but allow interactive manipulation on conventional computer 


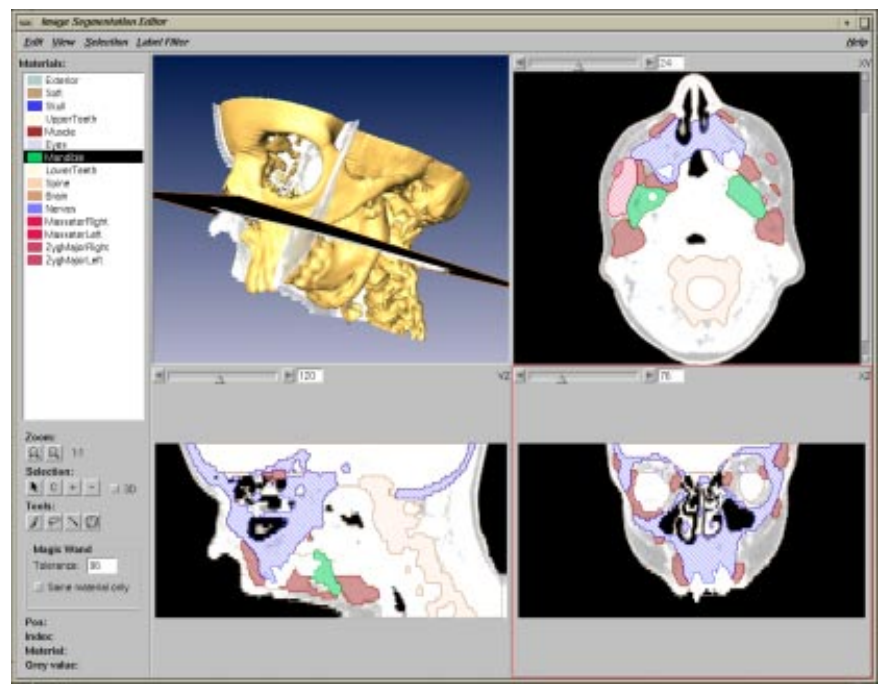

Fig. 1. Segmentation in three projections including 3D model view

systems. From the surface model, describing all classified tissue regions, a tetrahedral grid is generated automatically using a 3D advancing front algorithm [19]. In view of a finite-element simulation of soft tissue deformation, the mesh quality has to be optimized in a final step [11]. Surface and grid model are the basis of all subsequent planning (Fig. 2).
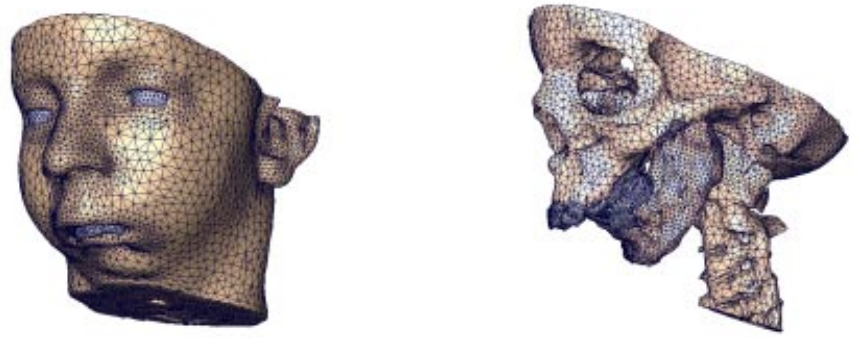

Fig. 2. 3D patient model with local adaptive resolution

\subsection{Diagnosis and Planning}

Two- and three-dimensional visualization techniques allow a multifarious interpretation of tomographic image data for diagnostic purposes. Currently the following techniques are used with our planning environment, either separately or in combination: a) 2D 
views of orthogonal or oblique slices, b) lateral, frontal and transversal radiographic views using intensity projections, c) volume rendering and d) surface rendering including texture mapping (Fig. 3).

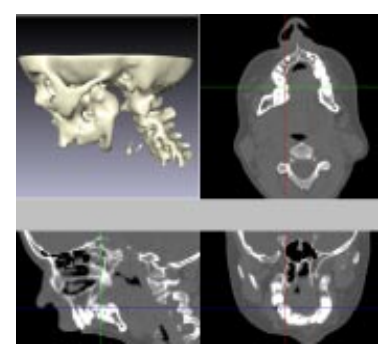

(a)

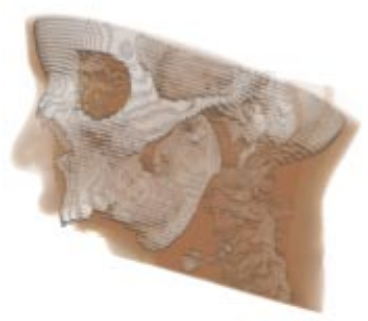

(c)

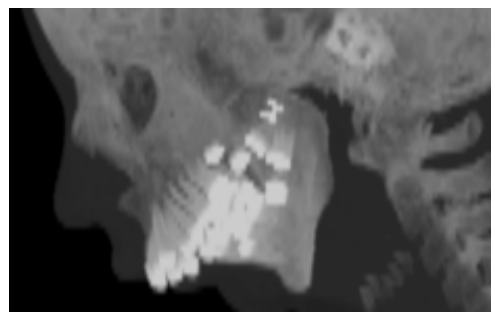

(b)

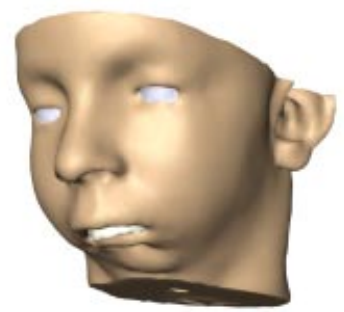

(d)

Fig. 3. 3D visualization for diagnosis and planning

The planning can be subdivided into three principal tasks: 1) cephalometric analysis, 2) osteotomy planning and 3) relocation of bony structures. Step 1 and 3 are usually repeated within a loop until an optimal functional rehabilitation is achieved. The 3D prognosis of the resulting soft tissue arrangement will give an additional and important planning criterion with respect to the aesthetic result.

\section{D Cephalometry}

For the quantitative assessment of maxillofacial dysmorphisms on a 3D model of the skull, orthodontic and orthognatic specifications have to be taken into account. The most important criteria are facial symmetry and dental occlusion. The combination of $2 \mathrm{D}$ profile analysis with $3 \mathrm{D}$ visualization techniques, under provision of $3 \mathrm{D}$ measuring tools for angles and distances [10], as well as methods for the comparison of pathologic and normative data [1, 15] will enhance the planning process tremendously. Therefore, methods of conventional 2D cephalometry have to be adopted to $3 \mathrm{D}$ and normative data as well as new paradigms for a $3 \mathrm{D}$ cephalometry have to be found. 
Currently, 3D measurements of distances and angles are implemented with our prototype system, as well as the visualization of 3D models in combination with planning planes, like profile projection, occlusional plane, medial plane or the eye-ear plane. Distances can be generally measured either in a projective, euclidean or geodaesic way.

\section{Osteotomy Planning}

Osteotomy planning depends on the patient specific situation as well as on standard procedures in maxillofacial surgery. For a 3D planning, arbitrarily shaped cut paths have to be defined, whereas vulnerable structures have to be preserved. Two different approaches are conceivable: 1) Osteotomy lines can be directly drawn onto the surface model of the skull using a 'virtual pen' or 2) a 3D cut path can be defined using a 'virtual saw'. Both methods require 3D input devices, two-handed interaction techniques, collision detection and force feedback for an intuitive use. In both cases the result will be either a 3D cut plane or a cut volume (according to the thickness of the saw blade) that separates parts and eventually removes material from the model. Performing cuts on the raw image data is a simple operation, but always requires the generation of a new 3D model for further planning. Cutting the surface model or the tetrahedral grid is much more difficult to implement, but speeds up the planning process, so that different strategies can be taken into account. Having such a cut plane or cut volume, we are also able to consider vulnerable structures, that are not already segmented within the modeling stage, by placing it into the $3 \mathrm{D}$ image data or mapping these data onto the respective surface.

\section{Bone Rearrangement}

The relocation of bony structures can either occur interactively under visual control (pick and transform), or via specification of numeric values (distances and angles) derived from the cephalometric analysis. Both methods are implemented with our planning system. An automatic correction of malformations, e.g. via statistical analysis, requires 3D craniofacial reference data that are currently not available. As result of the bone rearrangement we obtain displacement vectors for all nodes of our 3D model. These displacements serve as boundary conditions for our finite-element analysis, where the resulting 3D soft tissue deformation will be approximated.

\subsection{Soft Tissue Prediction}

The prediction of the entire spatial arrangement of the facial soft tissue will give the planning physician an additional, very important criterion for the assessment of the aesthetic impact of bone relocation. One can differentiate between two modes of simulation: 1) Deformation at interactive speeds with simple geometric or spring-mass models, and 2) consequent physics based deformation, using an elastomechanical model of biological tissues, a volumetric discretization of soft tissue and a finite-element approximation. The latter has been successfully integrated into our planning system, and has been discussed in detail within [11, 13, 14]. For a residual error norm, i.e. the approximation accuracy, of $10^{-6}$, simulation speed is between 30 and $180 \mathrm{~s}$ for 100.000 - 500.000 tetrahedra, even on a conventional $500 \mathrm{MHz}$ Pentium PC with more than 256 MB RAM to avoid memory swap. 


\section{Results}

With our planning environment it is possible to generate adequate $3 \mathrm{D}$ patient models for surgery planning and soft tissue simulation. Graphical planning aids are integrated for simple cephalometric analysis. Osteotomy lines can be specified, and separated bone parts can be relocated according to the surgeon's guidelines. The bone rearrangement is the basis for the prediction of the patient's post-operative appearance as described above. For two clinical cases a complete planning including soft tissue simulation has been performed at our institute in a tele-collaboration with surgeons in Munich.
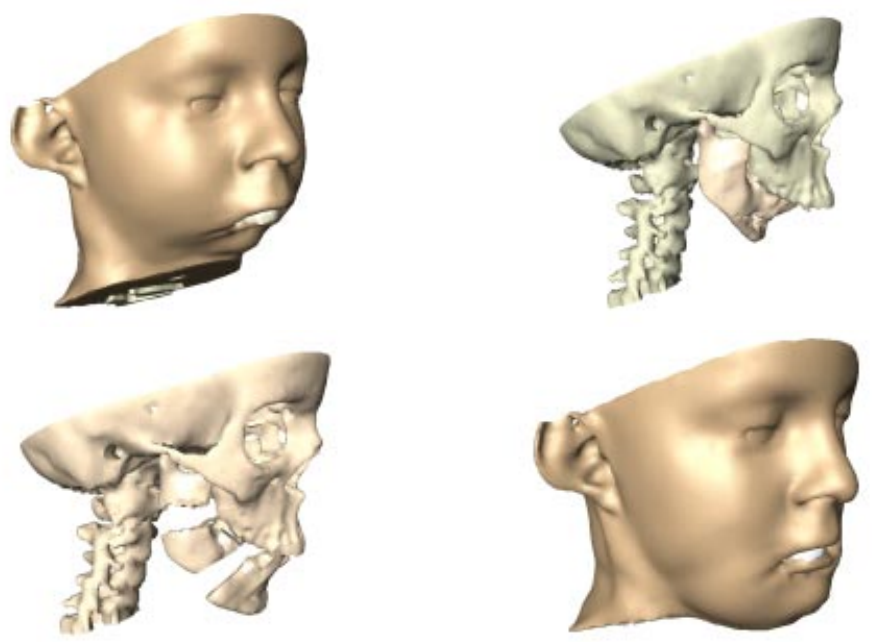

Fig. 4. 3D Planning of a bidirectional osteodistraction of the mandible: (top) preoperative situation, (bottom left) end position of the distractors, (bottom right) predicted facial appearance.

In the first case (Fig. 4) a bidirectional osteodistraction has been planned for a patient with a severe congenital mandibular hypoplasia. The rami mandibulae were separated horizontally and the corpus mandibulae has been separated on both sides with vertical cuts. The position of the simulated cuts do not exactly correspond to the surgical rules, and the mandibular nerve has not been addressed because in this case we were only interested in the post-operative appearance of the patient's face after reaching the end position of the distraction devices.

The initial surgical guidelines for the distraction vectors were: $30 \mathrm{~mm}$ in vertical direction (because of the limitation of available distraction devices), and $22 \mathrm{~mm}$ in horizontal direction. These displacements have been applied to the separated parts of the mandible, visually assessed and modified with respect to the soft tissue implication. The maximum displacements (vector norm) for a visually pleasing result have been $34 \mathrm{~mm}$ in vertical and $24 \mathrm{~mm}$ in horizontal direction (Table 1). The reference coordinate sys- 
tem is centered in the patient's head and the axes are: $\mathrm{x}$ ) left-right, $\mathrm{y}$ ) posterior-anterior and z) cranial-caudal.

Table 1. Distraction vectors and angles of all relocated bone parts

\begin{tabular}{|c|c|c|c|rrr|c|}
\hline bone part & \multicolumn{6}{|l|}{ translation in $\mathrm{mm}(\mathrm{LR} / \mathrm{PA} / \mathrm{CC})$} & \multicolumn{3}{|c|}{ rotational axis (LR/PA/CC) } & angle in \\
\hline ramus right & 1,50 & $-3,13$ & 4,88 & $-0,93$ & 0,02 & 0,34 & 2,24 \\
left & 0,02 & $-0,52$ & 1,34 & $-1,00$ & $-0,07$ & 0,00 & 0,48 \\
corpus right & 2,48 & $-8,19$ & 16,99 & $-1,00$ & 0,02 & 0,07 & 14,47 \\
left & 3,77 & $-2,65$ & 23,92 & $-1,00$ & $-0,08$ & $-0,01$ & 21,04 \\
front & $-6,22$ & 1,97 & 33,84 & $-1,00$ & 0,06 & $-0,14$ & 29,99 \\
\hline
\end{tabular}

In the second case (Fig. 5) for a patient with maxillary retrognathism and mandibular prognathism a bimaxillary operation has been planned, i.e. a high Le Fort I osteotomy of the maxilla, according to Bell and a sagittal split osteotomy on both sides of the mandible, according to Obwegeser-Dal Pont. The surgical guidelines were obeyed and the mandibular as well as the infraorbital nerve have been taken into account. The surgical guidelines for the bone transposition were: Advancement of the maxilla by $10 \mathrm{~mm}$ and back relocation of the mandible by $12 \mathrm{~mm}$. The visual assessment lead to a correction of the advancement to only $7 \mathrm{~mm}$, due to an over accentuated cheek and nose. However, the simulated results had no influence on the operation itself, but will be used for retrospective validation.

\section{Conclusion}

The quantification of the exact bone displacements as well as the validation of the simulated soft tissue deformation is still open. For the first case a second operation is scheduled in summer, where the horizontal distraction will be prepared. The availability of post-operative CT data will enable us to retrospectively simulate the same patient again, using the initial model but applying the real distraction values. Thus we are able to compare our simulation with the post-operative results qualitatively and quantitatively. A quantification of the difference between simulated and real soft tissue arrangement allows an inverse determination of the elasticity parameters, lying in a wide range for different tissue types (Poisson's Ratio $\nu \in[0.3,0.49]$ and Young's modulus $E \in[2,150]$ $\mathrm{kPa})$ [20].

We believe that 3D soft tissue prediction on basis of a volumetric discretization of a patient's head and FE methods has the potential for being an important criterion in surgery planning. A simulation time of a few minutes is neglectable in relation to the time needed for pre-operative planning. The most time consuming task is the segmentation, currently taking several hours. However, the segmentation result can be directly exported in STL format for the production of a stereolithographic model, thus conven- 

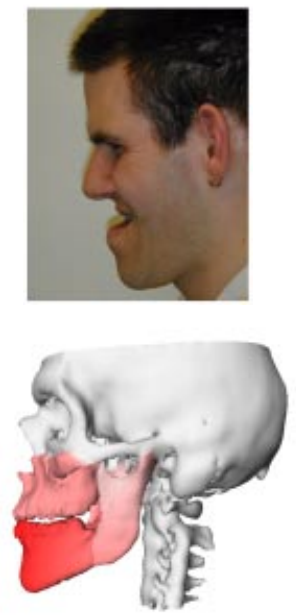
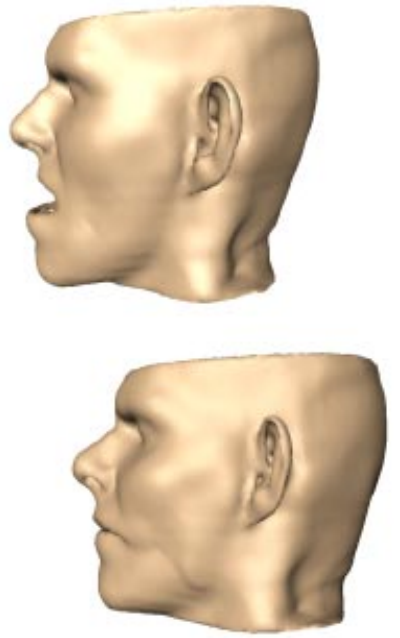
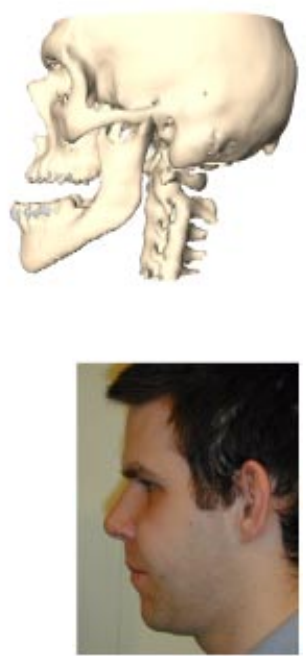

Fig. 5. 3D Planning of a bimaxillary osteotomy: (top) pre-operative situation, (bottom left) relocation of maxilla and mandible, (center) predicted facial appearance, (right) postoperative result

tional planning is still supported to assess different strategies in a non-destructive way, before applying the optimal one to a life size replica of the patient's skull.

\section{References}

[1] Cutting, C. ; Bookstein, F.L. ; Grayson, B. et'al.: Three-Dimensional Computer-Assisted Design of Craniofacial Surgical Procedures: Optimization and Interaction with Cephalometric and CT-Based Models. J. Plast. Reconstr. Surg. 77(6), pp. 877-885 (1986)

[2] Yasuda, T. ; Hashimoto, Y. ; Yokoi, S. and Toriwaki, J.-J.: Computer System for Craniofacial Surgical Planning Based on CT Images. IEEE Trans. Med. Imag. 9(3), pp. 270-280 (1990)

[3] Pieper, S.: CAPS: Computer Aided Plastic Surgery. Ph.D. thesis, MIT (1991)

[4] Altobelli, D.E. ; Kikinis, R. ; Mulliken, J.B. et al.: Computer-assisted three-dimensional planning in craniofacial surgery. J. Plast. Reconstr. Surg., Sep 92(4), pp. 576-587 (1993)

[5] Girod, S. ; Keeve, E. ; Girod, B.: Advances in interactive craniofacial surgery planning by $3 D$ simulation and visualization. Int. J. Oral Maxillof. Surg. 24(1), pp 120-125 (1995)

[6] Koch, R.M. ; Gross, M.H. ; Carls, F.R. et al.: Simulating Facial Surgery Using Finite Element Models. Computer Graphics, Proc. ACM Siggraph, pp. 421-428 (1996)

[7] Teschner, M.: Direct Computation of Soft-Tissue Deformation in Craniofacial Surgery Simulation. Ph.D. thesis, Friedrich-Alexander-Universität Erlangen-Nürnberg (2000)

[8] Schutyser, F. ; Van Cleynenbreugel, J. ; Ferrant, M. et al.: Image-Based 3D Planning of Maxillofacial Distraction Procedures Including Soft Tissue Implications. In: Delp, S.L. et al. (eds.) Medical Image Computing and Computer-Assisted Intervention (MICCAI), pp. 999-1007 (2000) 
[9] Everett, P.C. ; Seldin, E.B. ; Troulis, M. et al.: A 3-D System for Planning and Simulating Minimally-Invasive Distraction Osteogenesis of the Facial Skeleton. In: Delp, S.L. et al. (eds.) Medical Image Computing and Computer-Assisted Intervention (MICCAI), pp. 1029-1039 (2000)

[10] Bettega, G. ; Payan, Y. ; Mollard, B. et al.: A simulator for maxillofacial surgery integrating $3 D$ cephalometry and orthodontia. J. Comp. Aid. Surg. 5(3), pp. 156-165 (2000)

[11] Zachow, S. ; Gladilin, E. ; Hege, H.-C. and Deuflhard, P.: Finite-Element Simulation of Soft Tissue Deformation. In: Lemke, H.U. et al. (eds.): Computer Assisted Radiology and Surgery, pp. 23-28 (2000)

[12] Stalling, D. ; Hege, H.C. ; Zöckler, M. et. al.: Amira - An Advanced 3D Visualization and Modeling System, URL: http://amira.zib.de

[13] Gladilin, E. ; Zachow, S. ; Deuflhard, P. and Hege, H.-C.: Validation of a Linear Elastic Model for Soft Tissue Prediction in Craniofacial Surgery. SPIE Medical Imaging, San Diego, (2001)

[14] Gladilin, E. ; Zachow, S. ; Deuflhard, P. and Hege, H.-C.: A Biomechanical Model for Soft Tissue Simulation in Craniofacial Surgery. Medical Imaging and Augmented Reality, Hong Kong, China (2001)

[15] Brief, J. ; Hassfeld, S. ; Däuber, S. et al.: 3D Norm Data: The first step towards Semiautomatic Virtual Craniofacial Surgery. J. Comp. Aid. Surg. 5(3), pp. 353-358 (2000)

[16] Zachow, S. ; Lueth, T.C. ; Stalling, D. et al.: Optimized Arrangement of Osseointegrated Implants: A Surgical Planning System for the Fixation of Facial Prostheses. In: Lemke, H.U. et al. (eds.): Computer Assisted Radiology and Surgery (CARS), pp. 942-946 (1999)

[17] Hege, H.C. ; Seebaß, M. ; Stalling, D. ; Zöckler, M.: A Generalized Marching Cubes Algorithm Based On Non-Binary Classifications. ZIB Preprint SC-97-05 (1997)

[18] Stalling, D. ; Zöckler, M. ; Hege, H.-C.: Interactive Segmentation of 3D Medical Images with Subvoxel Accuracy. In: Lemke, H.U. et al. (eds.), Computer Assisted Radiology and Surgery, pp. 137-142 (1989)

[19] Jin, H. and Tanner, R.I.: Generation of Unstructured Tetrahedral Meshes by Advancing Front Technique. Int. J. Numer. Methods Eng. 36, pp. 1805-1823 (1993)

[20] Duck, F.A.: Physical Properties of Tissue - A Comprehensive Reference Book. Academic Press, Chap. 5, pp. 151 ff. (1990) 